\title{
Rapid-REM: A MATLAB simulator of the replaced-elements model
}

\author{
Holger SCHULtheis \\ Universität Bremen, Bremen, Germany
}

AND

Anna Thorwart and Harald Lachnit

Philipps-Universität Marburg, Marburg, Germany

\begin{abstract}
A recent proposal for an elemental account of associative learning phenomena is the replaced-elements model (REM) put forward by Wagner (2003). Although the ideas underlying this model are comparatively simple, implementation of the model is rather complex. In this article, we present Rapid-REM, a MATLAB simulator of Wagner's model. Rapid-REM features a graphical user interface for manipulating all essential parameter values and for control of the simulation process, graphical visualization of the simulation course and the results, and the alternative possibility of simulating the replaced-elements model as it was originally proposed (Wagner \& Brandon, 2001). Rapid-REM is available free of charge from www.staff.uni-marburg.de/ lachnit/Rapid-REM/. This simulator makes it easy to derive predictions for REM and evaluate them, and it will therefore facilitate insights into the mechanisms of associative learning.
\end{abstract}

Every organism in nearly every environment is faced with a multifaceted stream of stimuli that might be related to specific effects or outcomes. Associative learning theories are concerned with establishing how the various stimuli that occur in compounds are represented in the brain and how these representations enter into association with outcomes.

Pearce $(1987,1994,2002)$ assumes that stimuli are always processed configurally. In this configural theory, the elements of a compound collectively enter into a single association with one outcome. In this way, the model can straightforwardly account for the acquisition of configural discrimination problems, such as negative patterning. In a negative-patterning discrimination, conditioned stimuli (CSs) A and B are followed by an unconditioned stimulus (US) when they are presented alone, but not when they are presented together $(\mathrm{A}+, \mathrm{B}+, \mathrm{AB}-) . \mathrm{A}+$ and $\mathrm{B}+$ trials each result in the development of an association between the configural unit $\mathrm{A}$ and the US and between the configural unit $\mathrm{B}$ and the US, respectively, whereas $\mathrm{AB}-$ trials result in the development of an association between a configural unit $\mathrm{AB}$ and the US.

On the other hand, so-called elemental theories assume that each element of a compound enters into an association with the outcome. The widespread and influential Rescorla-Wagner theory (Rescorla \& Wagner, 1972) is an example of this view. Here, each CS is processed separately and develops its own associative link with the US. The change in associative strength is governed by the difference between the maximal associative strength sup- ported by a US $(\lambda)$ and the algebraic sum of the associative strengths of all the stimuli present in the current trial. The idea that the overall associative strength of a compound is based on the algebraic sum of the associative strengths of its elements is incorporated into many theories of associative learning (e.g., Mackintosh, 1975; Pearce \& Hall, 1980; Rescorla \& Wagner, 1972; Wagner, 1981). This simple version of the Rescorla-Wagner model, however, is not able to solve a number of discrimination learning problems, including negative patterning. A modified version of the Rescorla-Wagner model (Wagner \& Rescorla, 1972), however, is able to deal with the fact that different species are able to solve negative patterning; it does so by proposing the formation of a unique cue as an additional element when $\mathrm{A}$ and $\mathrm{B}$ are presented together. This unique cue allows different responding to $\mathrm{A}$ and $\mathrm{B}$ when they occur together than when they are presented alone, because the unique cue becomes associated with the absence of food, and thus is able to counteract the prediction of food on the basis of A and B.

Wagner and Brandon (2001) assumed that the representation of any isolable stimulus (e.g., CS A) consists of a collection of theoretical elements or nodes, each of which can get associated with the outcome. The changes in the strength of these associations are assumed to be determined by the Rescorla-Wagner (1972) equation. One group of nodes will be activated whenever their corresponding stimulus is presented. The instantiation of another group of nodes, however, depends on the presence of another stimulus. These nodes will be active only

H. Schultheis, schulth@informatik.uni-bremen.de 
when a stimulus is presented in conjunction with this other stimulus. Thus, according to this view, the representation of a compound involves the addition of elements (added-elements model). The unique-cue expansion of the Rescorla-Wagner model mentioned above can easily be described in terms of these context-dependent nodes.

The instantiation of a further group of nodes, however, depends on the absence of other stimuli. These nodes are activated only when a specific stimulus is presented alone, and they are inhibited when the corresponding stimulus is shown as part of a compound (inhibited-elements model). In this manner, the inhibited-elements model assumes that the presence of one stimulus alters the representation of another stimulus within this context, as compared with when a stimulus is presented alone.

In their model, Wagner and Brandon (2001) combined both ways of thinking (added and inhibited) to yield the first replaced-elements model (REM). This conception makes a simple assumption about the replacement occurring in different contexts. If a CS A can be presented alone or in either or both of two unrelated contexts, with CS B or $\mathrm{C}$, the context-dependent nodes of $\mathrm{A}$ that are replaced by occurrence in Context B are completely different from those replaced by occurrence in Context C. This, however, implies that the proportion of elements replaced by a context shift (among the many possible ones) has to be small.

In order to circumvent this restriction, Wagner (2003) suggested a modification of the REM by specifying a replacement parameter $r$ (ranging from 0 to 1 ) to determine the exact proportion of nodes that are replaced and/or inhibited by the addition or removal of stimuli from a compound. In this model, the replacements resulting from the two contextual manipulations are statistically independent. With $r=0$, the REM becomes purely linear, equivalent to the Rescorla-Wagner (1972) model, and with $r=1$, it becomes a purely configural model. Contrary to Pearce's $(1987,1994,2002)$ configural theory, however, with $r=1$ there is no generalization at all, because the different compounds now do not have any elements in common.

Configural theories account for the phenomena of learning in terms of configural representations, whereas elemental theories do so in terms of elemental representations. Although there has been a rich and productive history of research on the elemental/configural (or part/whole) distinction, this issue is not settled yet, for the results of different experiments support and contradict each view. Furthermore, the necessity of configural representations per se, as in Pearce's configural theory, was fundamentally questioned when Wagner and Brandon (2001) theoretically showed that under certain conditions, Pearce's configural theory is isomorphic with an inhibited-elements model, which by itself is purely elemental.

Evidence in support of the REM has been obtained by Brandon, Vogel, and Wagner (2000) and Glautier (2004). Since simulation of the predictions of this model is rather difficult, Glautier (2007) described a method to generate the correct stimulus representations. These representations can then be used in standard simulation programs of associative learning theories. Yet, applying the suggestions of
Glautier (2007) still requires considerable programming expertise and work, and thus, REM simulations might not be easily available for researchers working on associative learning theories.

In the present article, we offer an intuitively operated program, called Rapid-REM because it eases and quickens REM simulation, to compute the predictions of the revised version of the REM (Wagner, 2003). This software allows the specification of all relevant parameters of the simulation via a graphical user interface (GUI) and specification of the to-be-presented CS in a straightforward manner, without having to bother with the precise CS representations. Rapid-REM takes care of setting up the necessary CS representation in terms of (proportions of) elements, the simulation of the learning process, and a graphical presentation of the learning process and outcome. As an additional feature, it is also possible to simulate the original version of the REM proposed by Wagner and Brandon (2001) with Rapid-REM. In the following section, we will describe Rapid-REM's components and use in more detail.

\section{RAPID-REM}

Rapid-REM is written in MATLAB and has been tested with MATLAB 6.5 and higher, on both Windows and Linux operating systems. The software package can be obtained in its most recent version (currently, 2.3) free of charge from www.staff.uni-marburg.de/ lachnit/RapidREM/. Since Rapid-REM simulates the two different REM versions in rather similar ways, we will first describe the operation of Rapid-REM for the newer, modified version of the REM, and subsequently highlight those aspects that are different for simulating the original REM.

\section{Graphical User Interface}

Fields. The GUI of Rapid-REM (Rapidrem.m; see Figure 1A) comprises 14 fields for parameterizing and six buttons for controlling the simulation. The fields - from left to right and top to bottom in the figure - are listed in this section.

File fields. The first four fields allow specifying the full path of different files relevant for the simulation. For each field, one can either specify the path by hand or use the "Search" button on the right to pick the desired file. The first field ("Pattern file") holds the path to the file containing the definitions for the CSs (compounds) presented throughout the to-be-simulated associative learning setting. By default, Rapid-REM assumes that pattern files have the ending ".pat." The second field ("Test file") holds the path to the file containing the definitions for the CSs (compounds) for which responding should be evaluated after learning. Test files have the default ending ".tst." The last two fields ("Weight output file" and "Weight input file") have been included in the GUI to allow for saving and restoring already learned associative weights. Files storing weights have the default ending ".net." Whereas the pattern and test files must be specified to run the simulator, indicating weight files is optional. The precise structure of all file types will be discussed in detail below. 
A

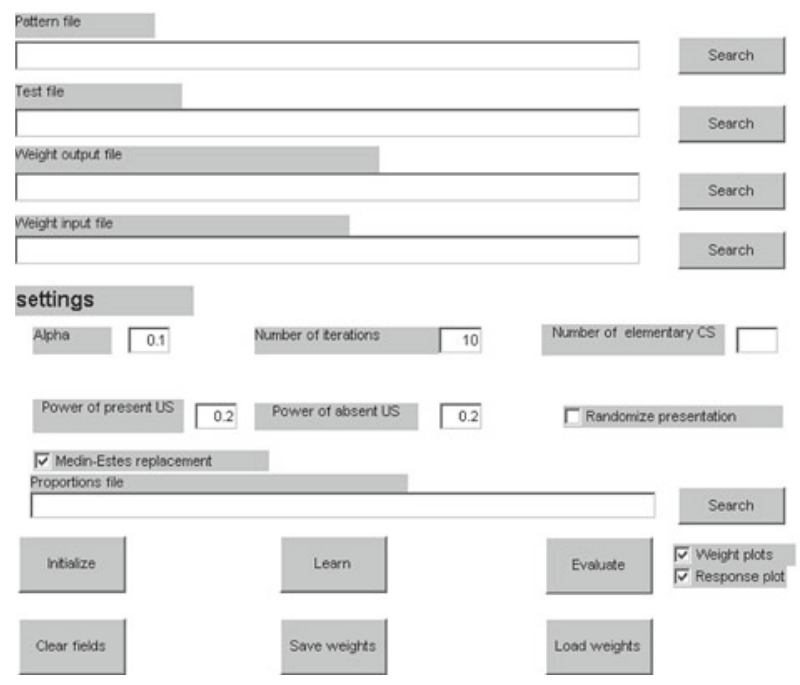

B

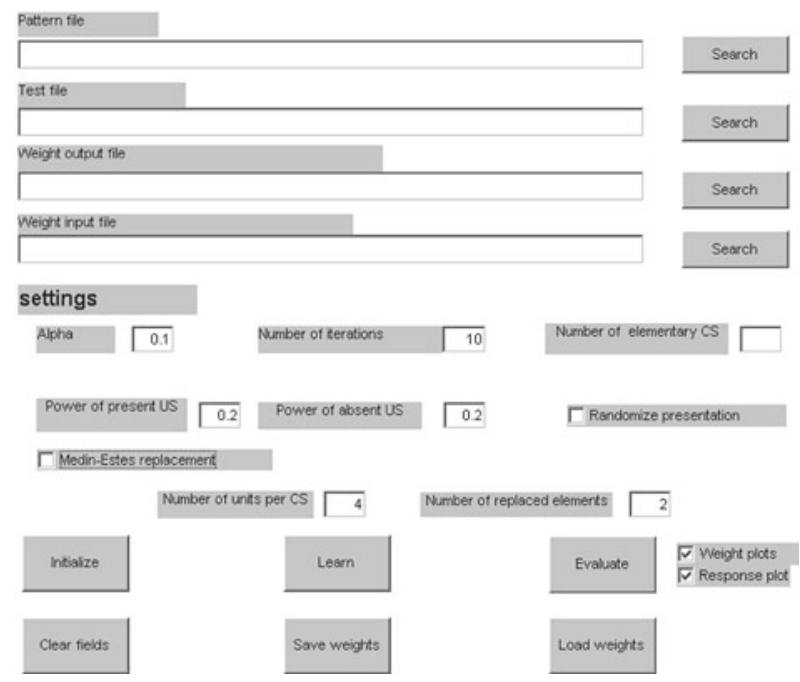

Figure 1. The graphical user interface (GUI) of Rapid-REM: (A) GUI of the current REM version; (B) GUI of the original REM version.

Alpha. This field allows setting of the learning rate for the Rescorla-Wagner learning rule. The default value for the learning rate is 0.1 .

Number of iterations. The number in this field controls how often the CSs (and compounds) specified in the pattern file are presented during learning. In particular, all CSs (and compounds) given will be presented for each single iteration. Thus, if one simulates a setting with 7 different configurations of CSs and 100 iterations, the simulation will comprise $7 * 100$ presentations of CS configurations and the corresponding weight updates. The default value for the number of iterations is 10 .

Number of elementary CS. This field holds the number of different elementary CSs employed in the setting to be simulated. In the case of negative patterning $(\mathrm{A}+, \mathrm{B}+$, $\mathrm{AB}-$ ), for instance, the number of elementary $\mathrm{CSs}$ is two (A and $\mathrm{B})$.

Power of present US. This field allows parameterization of the power of the present US. This parameter corresponds to $\beta$ in the Rescorla-Wagner learning rule. The default value for this power is also 0.2 .

Power of absent US. Here the power of an absent US can be parameterized. This parameter corresponds to $\beta$ in the Rescorla-Wagner learning rule. The default value for this power is also 0.2 .

Randomize presentation. In the pattern file, the CS configurations necessarily have to be listed in a fixed order. However, it might be desirable to have the different CS configurations presented in a randomized order in each iteration. If this checkbox is checked, presentation order will be randomized. If this box is unchecked, in each iteration the simulation will present the CS configurations in the order given in the file. By default, randomized presentation is used.

Medin-Estes replacement. This checkbox determines whether the modified or original REM will be simulated.
When it is checked (which is the default), the modified version of the REM (Wagner, 2003) will be simulated.

Proportions file. This field is only available when the modified REM is simulated. It specifies the full path (which can be either explicitly typed in or chosen using the "Search" button) of the file containing the proportions of replaced $(r)$ and not replaced $(1-r)$ elements of any elementary CS, in the context of any other elementary CS involved in the investigated learning setting (see above). The precise structure of this file will be explicated below. Simulation of the modified REM is not possible without this file.

Weight plots. As detailed below, Rapid-REM features graphical display of the weight course during learning and of the final weights resulting from learning. Since the graphical display of the weight information might not always be desired, this check box allows the user to selectively disable and enable the graphical weight output.

Response plot. Analogous to the "Weight plots" check box, this check box controls whether the course and final result of the simulated conditioned responses will be graphically displayed or not.

Buttons. After all relevant file locations and parameter values have been specified, the simulation can be started and controlled by the six buttons at the bottom of the GUI. The functions of the control buttons are detailed below.

Initialize. Pressing this button will initiate a check of whether all necessary information has been specified. If this is the case, all relevant files and fields will be opened and read. On the basis of the information, the simulation will be set up by creating an appropriate stimulus representation for each trial specified in the pattern and test files. Details regarding the setup of the stimulus representations can be found in the Appendix. Initialization is a prerequisite for further simulations.

Learn. After the simulation has been initialized, learning can take place. Learning proceeds by sequentially pre- 
senting all CS configurations specified in the pattern file to the net and updating the weights accordingly (see the Appendix) for as many times as specified in the "Number of iterations" field (see above). Once started, learning cannot be interrupted, but will proceed until all iterations have been processed. Intermediate results for both responses and weights, however, will be registered and can later be inspected via the evaluation function.

Evaluate. Evaluation basically comprises the graphical display of the courses of the weights and the simulation responses registered during learning. Weights and responses are displayed in separate plots. In the weights plot (see Figure 2A), each weight of the simulation and its change during learning is displayed by a separate curve. Different curve signatures (i.e., different line styles and types of markers) are automatically generated for each individual weight. To reduce visual clutter in the case of a large number of weights, a maximum of six weights may be presented together in one figure. If the simulation comprises more than six weights, new figures for groups of six weights will automatically be created. The response plot (Figure 2B) displays the course of the strength of responding to each of the CS configurations specified in the test file. As with the weights, different curve signatures are automatically created for different CS configurations. The legend inside the figure helps identify the test case to which each curve belongs: "T $x$ " represents the CS configuration that is the $x$ th configuration specified in the test file. Currently, 12 different curve styles can be distinguished. Apart from the curve styles, however, an additional feature allows further discriminating between test cases. In the test file (see below), one may not only specify the CS configurations to test, but also whether excitation (CS+) or inhibition $\left(\mathrm{CS}_{0}\right.$ or CS-) of responses should occur (if, for example, CS A is consistently paired with the US during training, one should expect conditioned responding to A). Curves rep-

A

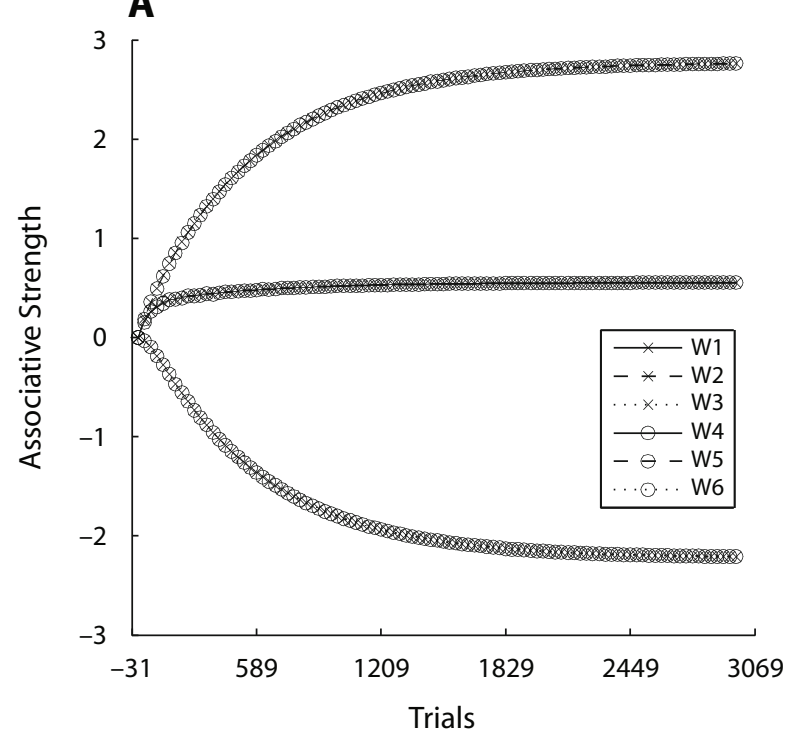

resenting CSs + will be drawn with bold lines, whereas curves representing CSs - will be drawn with a thin line. Although we tried to render the automatically generated graphical display as clear as possible, the standard output format might not be suitable for special-purpose analyses or needs. To facilitate special analyses, the registered weight and response courses are not only graphically displayed, but also written to two files. These files will be created in the directory where the pattern file of the current simulation is located. The files are named Weights.ver and Responses.ver for the weight and response courses, respectively. Furthermore, since generating the weight plots can be especially time-consuming, the user may deactivate the plots by unchecking the relevant check boxes (see above), whereas the files are always created.

Clear fields. This button deletes all text entries currently in the GUI fields; the check boxes are not affected.

Save weights. If a path is specified in the "Weight output file" field (see above), pressing this button will write the current weight values to the given file. Storing weights allows for archiving the current state of a simulation in order to, for example, continue learning later on, exchange learned nets with other researchers, or avoid repeated time-consuming computations for complex simulations.

Load weights. In cases in which (1) weights have been stored previously and (2) the corresponding file is given in the "Weight input file" field (see above), pressing this button will restore the saved weight values.

\section{Simulating the Original REM}

As already mentioned, Rapid-REM allows for simulating the REM not only in its current conception (Wagner, 2003 ), but also in the form in which it was originally proposed (Wagner \& Brandon, 2001). If the box "MedinEstes replacement" is unchecked, the original rather than the current version of REM will be simulated (see Fig-

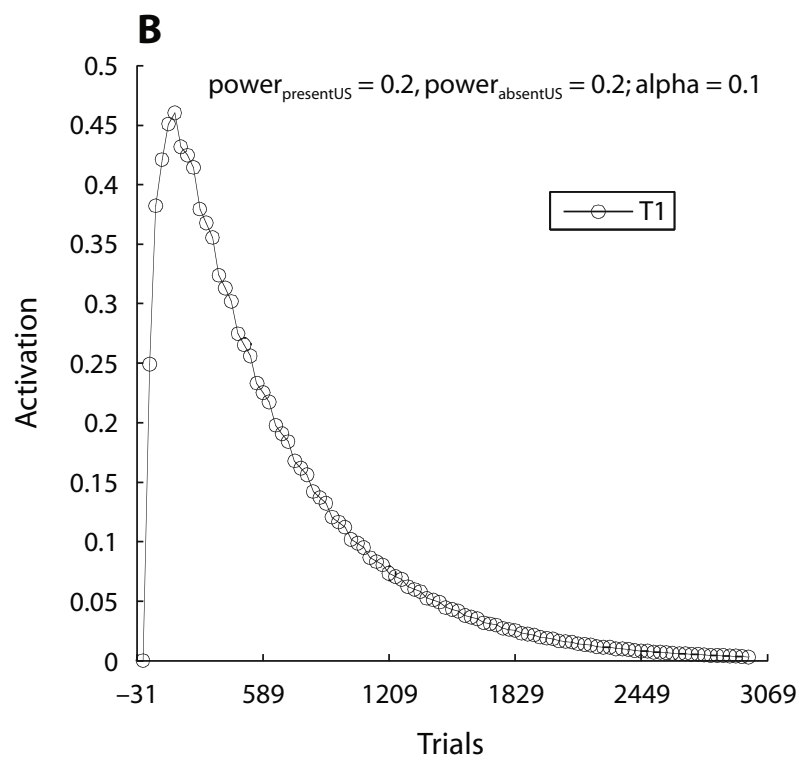

Figure 2. The courses of the simulated weights $(\mathrm{A})$ and responses $(\mathrm{B})$ for the negative-patterning problem $\mathrm{A}+, \mathrm{B}+, \mathrm{AB}-. \mathrm{Only}$ the response for $A B$ is shown. 
ure 1B). The main difference in use between these two types of simulations - apart from considerable changes in the internally employed representations of the CSs-pertains to the (specification of the) replacement parameters. When the "Medin-Estes replacement" box is unchecked, the field with the path to the proportions file is replaced by two new fields. The first of these fields ("Number of units per CS") holds the number of units utilized for each of the elementary CSs. The second field ("Number of replaced elements") gives the number of units of each elementary CS being replaced in the context of other CSs. Both of these fields are required to simulate the original REM. Obviously, the number of replaced elements should be smaller than the number of units per CS for the simulation to run properly.

\section{Files}

Rapid-REM draws on a number of files to realize the simulation. Since the simulation will only work properly if the files are structured correctly, we will shortly describe the correct formats for the different types of files in this section.

Pattern files start with a line holding a single integer. This integer specifies the number of CS configurations being listed in the file (i.e., the configurations that are to be employed during learning). The next line holds a sequence of $0 \mathrm{~s}$ and $1 \mathrm{~s}$ separated by white spaces. The length of the sequence should be identical to the number of elementary CSs employed. Each digit represents one of the elementary CSs; a 1 indicates that this CS is present, and a 0 indicates that it is absent. As a result, the complete sequence (i.e., the corresponding line in the file) represents one CS configuration. The next line again holds only a single integer, which can be a 1 or a 0 . A 0 indicates that during learning, the CS configuration represented by the preceding line is not paired with the US, and a 1 indicates that the CS configuration is paired with the US. Such pairs of a CS configuration with US presence can occur arbitrarily often in a pattern file, but the number of configuration-US presence pairs should equal the number given in the first line of the file. The pattern file for negative patterning $(\mathrm{A}+, \mathrm{B}+, \mathrm{AB}-)$, for instance, is shown in Figure 3A.

Test files are structurally identical to pattern files. They start with a single integer on the first line (specifying the number of CS configurations being listed in the file), followed by pairs of lines, one for each CS configuration. The first line of each pair of lines contains a specific CS configuration. The second line contains either a single 0 , if this configuration is expected to be a $\mathrm{CS}_{0}$ or $\mathrm{CS}-$, or a single 1 , if this configuration is expected to be a $\mathrm{CS}+$. Importantly, the CS configurations listed in the test file are completely independent of the configurations specified in the pattern file: The test file may contain more or fewer configurations than the pattern file, and they may be identical to or different from those in the pattern file. If one were only interested in the responses to $\mathrm{AB}$ in the negative-patterning setting described above, the test file would look like the one shown in Figure 3B.

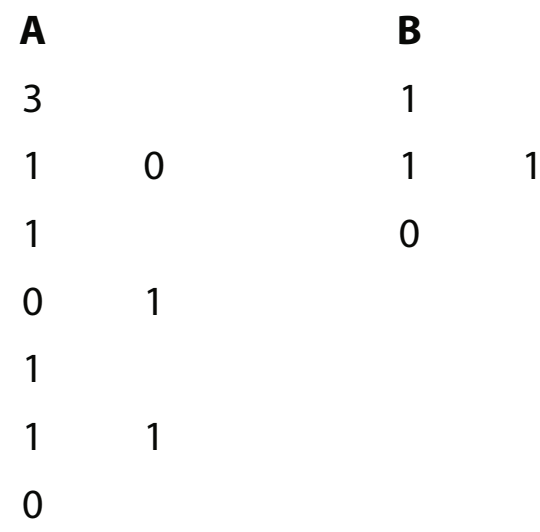

Figure 3. Examples of pattern (A) and test (B) files. See the text for details.

The proportions file contains $n$ lines, where $n$ is the number of elementary CSs. Line 1 represents the proportions for the first $\mathrm{CS}\left(\mathrm{CS}_{1}\right)$, line 2 those for the second $\mathrm{CS}$ $\left(\mathrm{CS}_{2}\right)$, and so on. Each of these lines again contains $n-1$ real numbers. For any line $L$, these numbers indicate the proportions of elements to be replaced for $\mathrm{CS}_{L}$ in the context of the other CSs. More precisely, $r_{j}^{i}$ (with $i$ the index of the line, $j$ the index of the column, and $i \neq j$ ) is the proportion of elements of $\mathrm{CS}_{i}$ that will be replaced in the presence of $\mathrm{CS}_{j}$. Thus, for each CS there is a line in the proportions file that holds the proportions of elements to be replaced in the presence of every other CS. Since the numbers in each line represent proportions of to-be-replaced elements, they should range within the closed interval $[0,1]$. One possible proportions file for the negative-patterning example above is shown in Figure 4A.

Basically, the weights file contains a list of current weight values, where the order of the weights corresponds to the order of the units representing CS elements (see the Appendix for details). Yet, there are two qualifications to this general principle. First, to increase the legibility of the file for humans, the list of weights is split into blocks of eight, each occupying a single line in the file. Second, the weights alone are not sufficient to reinitialize a previously trained network. For reinitialization, at least the number of weights and the number of CSs are also necessary. In the case of the original REM, the number of units per CS and number of replaced units are required in addition. As
A
0.2
6
0.2
2
\#Weights 1 to 6
$0.552 .76-2.210 .552 .76-2.21$

Figure 4. Examples of proportions (A) and weights (B) files. See the text for details. 
a result, the weights file has the following form: The first line holds the number of weights. The second holds - in this order - the number of CSs, the number of units per CS, and the number of replaced elements per CS. All following lines then contain - in blocks of eight - the weight values. Figure 4B shows a weights file stored after learning 10 iterations of the negative-patterning example above.

All files are plain text files. That is, any editor for ASCII text files can be used to create, edit, and save the files.

\section{Computational Complexity}

Generally, the algorithms realizing the REM simulation are computationally cheap with respect to both the memory space and computation time needed. Yet, because of the stimulus representation formalism required by the modified REM, simulations might last rather long in certain learning settings. This is because the number of units required to represent all CS configurations grows exponentially with the number $n$ of elementary CSs. More precisely, the number of units $N_{\mathrm{u}}$ is given by

$$
N_{\mathrm{u}}=1+n * \sum_{i}^{n-1}\left(\begin{array}{c}
n-1 \\
i
\end{array}\right) * 2^{i} .
$$

Consequently, whereas the situation of 5 elementary CSs, which requires only 405 units, is unspectacular, 10 elementary CSs will already require nearly 200,000 units to be simulated. Such large numbers of units might considerably tax even modern computing equipment-especially when the number of iterations to be performed is also high. Note that this exponential scaling is a property of the REM as proposed by Wagner (2003) and is not a specific shortcoming of Rapid-REM. In any case, experimental data on associative learning involving more than 5 elementary CSs seem to be scarce, and thus the exponential scaling seems, at least at the moment, to pose no serious practical problem.

\section{Standard Use and an Example}

Summing up, a standard simulation project using Rapid-REM will usually comprise the following steps:

1. Write the pattern file;

2. Write the test file;

3. Write the proportions file;

4. Fill all fields with the relevant parameter values;

5. Initialize the simulation;

6. Learn for as many iterations as desired, with possible occasional storage of intermediate weight values;

7. Evaluate the simulation results.

To illustrate the process further, consider again the negative-patterning example mentioned above. For this example, we must prepare the pattern file (Figure 3A), the test file (only testing the compound AB; Figure 3B), and the proportions file (Figure 4A). By inserting 2 in the "Number of elementary CS" field, inserting 1000 into the "Number of iterations" field, and using the default values for the rest of the parameters, the simulation preparations are finalized. By pressing "Initialize" and then the "Learn" button, the associative weights are changed. The resulting weights are stored (using the "Save weights" button) in the file learnedNet.net (Figure 4B). Finally, the results of the simulation and the course of weight and response changes can be visualized by pressing the "Evaluate" button. The resulting graphical displays are shown in Figure 2.

\section{CONCLUSION}

In order to explain and better understand the processes involved in associative learning, various theories and models have been proposed over the last few decades (e.g., Mackintosh, 1975; Pearce, 1987, 1994; Rescorla \& Wagner, 1972; Wagner, 2003; Wagner \& Brandon, 2001). Against the background of this multitude of models, the question arises, which of these theories can best account for the observed associative learning phenomena? The common way to approach this question is to derive behavioral predictions from the proposed models and compare the predictions to the actually occurring behavior (see, e.g., Lachnit, Schultheis, König, Üngör, \& Melchers, in press). Obviously, one prerequisite of this approach is the possibility of generating predictions of the different models for arbitrary associative learning settings. Whereas this is relatively straightforward for some of the models, such as the elemental theory of Rescorla and Wagner or the configural approach proposed by Pearce (1994), other models require considerable mathematical and programming expertise to derive predictions. A prominent example of the latter type of model is the new version of the REM (Wagner, 2003). Although the idea underlying the model is not too complicated, setting up the correct CS representations is hard. This has led Glautier (2007) to propose an algorithm that allows the generation of the correct CS representations for arbitrary situations. However, problems remain with Glautier's (2007) proposed algorithm: First, he presented it only in pseudocode - that is, implementation of the algorithm must still be carried out by anyone wanting to derive predictions for the REM. Second, setting up the stimulus representation is only one part of a full simulation of the REM, which in addition requires the handling of parameter values and presentation of the simulation results.

Here, we presented an intuitively operated REM simulator, called Rapid-REM, which not only takes care of setting up the CS representations, but also allows controlling the simulation and all its parameters via a GUI. For example, the course and results of a simulation can be made graphically available by a single buttonpress. In addition to the current version of the REM, Rapid-REM also allows simulation of the original REM proposed by Wagner and Brandon (2001). With Rapid-REM, opportunities for simulation and assessment of the REM are easily available; in this way, we hope that Rapid-REM will facilitate evaluating and comparing different associative learning theories, thereby helping gain a deeper understanding of the processes and representations involved in associative learning. 


\section{AUTHOR NOTE}

The research reported in this article was partially supported by a grant from the Deutsche Forschungsgemeinschaft to H.L. (Grant DFG La 564/10-4). Correspondence concerning this article should be addressed to H. Schultheis, Department of Informatics, Universität Bremen, Postfach 330440, 28334 Bremen, Germany (e-mail: schulth@, informatik.uni-bremen.de or lachnit@staff.uni-marburg.de).

\section{REFERENCES}

Brandon, S. E., Vogel, E. H., \& Wagner, A. R. (2000). A componential view of configural cues in generalization and discrimination in Pavlovian conditioning. Behavioural Brain Research, 110, 67-72.

GlautieR, S. (2004). Asymmetry of generalization decrement in causal learning. Quarterly Journal of Experimental Psychology, 57B, 315-329.

Glautier, S. (2007). Simulation of associative learning with the replaced elements model. Behavior Research Methods, 39, 993-1000.

Lachnit, H., Schultheis, H., König, S., Üngör, M., \& Melchers, K. G. (in press). Comparing elemental and configural associative theories in human causal learning: A case for attention. Journal of Experimental Psychology: Animal Behavior Processes.

Mackintosh, N. J. (1975). A theory of attention: Variations in the associability of stimuli with reinforcement. Psychological Review, 82, 276-298

Pearce, J. M. (1987). A model for stimulus generalization in Pavlovian conditioning. Psychological Review, 94, 61-73.
Pearce, J. M. (1994). Similarity and discrimination: A selective review and a connectionist model. Psychological Review, 101, 587-607.

Pearce, J. M. (2002). Evaluation and development of a connectionist theory of configural learning. Animal Learning \& Behavior, 30, 73-95.

Pearce, J. M., \& Hall, G. (1980). A model for Pavlovian learning: Variations in the effectiveness of conditioned but not of unconditioned stimuli. Psychological Review, 87, 532-552.

Rescorla, R. A., \& Wagner, A. R. (1972). A theory of Pavlovian conditioning: Variations in the effectiveness of reinforcement and nonreinforcement. In A. H. Black \& W. F. Prokasy (Eds.), Classical conditioning II: Current research and theory (pp. 64-99). New York: Appleton-Century-Crofts.

WAGNER, A. R. (1981). SOP: A model of automatic memory processing in animal behavior. In N. E. Spear \& R. R. Miller (Eds.), Information processing in animals: Memory mechanisms (pp. 5-47). Hillsdale, NJ: Erlbaum.

WaGner, A. R. (2003). Context-sensitive elemental theory. Quarterly Journal of Experimental Psychology, 56B, 7-29.

WAGNER, A. R., \& BRANDON, S. E. (2001). A componential theory of Pavlovian conditioning. In R. R. Mowrer \& S. B. Klein (Eds.), Handbook of contemporary learning theories (pp. 23-64). Mahwah, NJ: Erlbaum.

WAGNer, A. R., \& Rescorla, R. A. (1972). Inhibition in Pavlovian conditioning: Application of a theory. In R. A. Boakes \& M. S. Halliday (Eds.), Inhibition and learning (pp. 301-340). London: Academic Press.

\section{APPENDIX}

Although the algorithms underlying Rapid-REM are not the main focus of this article, a brief description of the general procedure seems in order, to give the reader a more thorough understanding of the workings of the simulator. In particular, this will facilitate the interpretation of the stored weights (see, e.g., Figure 4B).

Similar to Glautier (2007), we use single elements to represent subsets of elements of each of the simple CSs involved in some discrimination problem. In the negative-patterning problem $\mathrm{A}+, \mathrm{B}+, \mathrm{AB}-$, for instance, the elements of each of the simple CSs (A, B) are represented by three units. The first of these units represents the subset of elements activated whenever $\mathrm{A}$ is present, the second the subset of elements activated when $\mathrm{A}$ is presented without $\mathrm{B}$, and the third the subset of elements when $\mathrm{A}$ is presented in compound with $\mathrm{B}$. The three units for B are arranged analogously, resulting in an overall array of six units, the first three representing subsets of elements of A and the last three representing subsets of elements of B. Each of the units used has a connection to the unit representing the US. The weights represent the strength of these connections, and they are written to the corresponding file (see, e.g., Figure 4B) in the same order the units are arranged in. This coding scheme can be and has been adapted straightforwardly to other discrimination problems, as described in Glautier (2007).

For each trial during learning, the units are activated according to the CS present on that trial. If, for example, only A is presented on a trial, only the first two units will be activated, whereas if A and B are presented in compound, Units 1, 3, 4, and 6 are activated. Since the units represent subsets (i.e., proportions) of elements of simple CSs, the amount of their activation is proportional to the number of elements they represent. More precisely, each unit's activation is identical to the proportion of elements it represents (see Glautier, 2007, for an in-depth discussion of how the proportions for the different units are computed).

As a result, in Rapid-REM the simulated conditioned responses CR are computed as

$$
\mathrm{CR}=\sum_{i} a_{i} w_{i}
$$

where $w_{i}$ is the weight of the connection from unit $i$ to the US, and $a_{i}$ is the proportion of elements represented by unit $i$ if this unit is activated or 0 if the unit is not activated. Updating of the weights occurs only for units activated on a trial. For these units, the weight update $\Delta w$ is computed as

$$
\Delta w=\alpha \beta(\lambda-\mathrm{CR}) .
$$

